\title{
On the estimation and detection of the Rees-Sciama effect
}

\author{
M. J. Fullana, ${ }^{1 \star}$ J. V. Arnau, ${ }^{2}$ R. J. Thacker, ${ }^{3 \star}$ H. M. P. Couchman ${ }^{4}$ and D. Sáez ${ }^{5,6 \star ~}$ \\ ${ }^{1}$ Institut de Matemàtica Multidisciplinar, Universitat Politècnica de València., E-46022 València, Spain \\ ${ }^{2}$ Departament de Matemàtica Aplicada, Universitat de València., E-46100 Burjassot, Valencia, Spain \\ ${ }^{3}$ Department of Astronomy and Physics, Saint Mary's University, Halifax, NS B3H 3C3, Canada \\ ${ }^{4}$ Department of Physics and Astronomy, McMaster University, 1280 Main St. West, Hamilton, ON L8S 4M1, Canada \\ ${ }^{5}$ Departament d'Astronomia i Astrofísica, Universitat de València., E-46100 Burjassot, València, Spain \\ ${ }^{6}$ Observatori Astronòmic, Universitat de València, E-46980 Paterna, Valencia, Spain
}

Accepted 2016 October 10. Received 2016 October 1; in original form 2016 July 28; Editorial Decision 2016 October 6

\begin{abstract}
Maps of the Rees-Sciama (RS) effect are simulated using the parallel $N$-body code, HYDRA, and a run-time ray-tracing procedure. A method designed for the analysis of small, square cosmic microwave background (CMB) maps is applied to our RS maps. Each of these techniques has been tested and successfully applied in previous papers. Within a range of angular scales, our estimate of the RS angular power spectrum due to variations in the peculiar gravitational potential on scales smaller than $42 / \mathrm{h}$ megaparsecs is shown to be robust. An exhaustive study of the redshifts and spatial scales relevant for the production of RS anisotropy is developed for the first time. Results from this study demonstrate that (i) to estimate the full integrated RS effect, the initial redshift for the calculations (integration) must be greater than 25, (ii) the effect produced by strongly non-linear structures is very small and peaks at angular scales close to 4.3 arcmin, and (iii) the RS anisotropy cannot be detected either directly-in temperature CMB maps - or by looking for cross-correlations between these maps and tracers of the dark matter distribution. To estimate the RS effect produced by scales larger than $42 / \mathrm{h}$ megaparsecs, where the density contrast is not strongly non-linear, high accuracy $N$-body simulations appear unnecessary. Simulations based on approximations such as the Zel'dovich approximation and adhesion prescriptions, for example, may be adequate. These results can be used to guide the design of future RS simulations.
\end{abstract}

Key words: methods: numerical - cosmic background radiation - cosmology: theory - largescale structure of Universe.

\section{INTRODUCTION}

In this paper, we revisit the so-called Rees-Sciama (RS; Rees \& Sciama 1968) effect. In Puchades et al. (2006, hereafter paper I), the effect was properly defined and its angular power spectrum estimated using various numerical codes and computational methods. Simulations of cosmic structures were performed with a ParticleMesh (PM) $N$-body code (Hockney \& Eastwood 1988). In Cai et al. (2010), numerical techniques were designed, tested, and applied to estimate the RS angular power spectrum for angular scales, $\ell$, $<10^{3}$. Our simulations and numerical methods allow us to estimate this spectrum for $\ell>10^{3}$. Combined, these procedures should lead to accurate estimated RS spectra for $\ell<3.2 \times 10^{3}$. Our methods allow us to probe the spatial and temporal scales that contribute to the RS angular power spectra in contemporary cosmologies.

For the large simulation boxes required in our study (see below), the spatial resolution of PM codes is a limitation, in particular, in

^E-mail: mfullana@mat.upv.es (MJF); thacker@ap.stmarys.ca (RJT); diego.saez@uv.es (DS) regions with large non-linear excesses of dark matter as well as in almost empty voids. Thus, better $N$-body simulations are necessary to get more accurate estimates of the RS angular power spectrum, the spatial scales producing the effect, and so forth. These results are necessary in order to assess the possibility of detecting the RS contribution to the cosmic microwave background (CMB) anisotropy for $\ell>10^{3}$. To address these concerns, we use the parallel HYDRA implementation of the Adaptive Particle-Particle-Particle-Mesh (AP3M) code (Thacker \& Couchman 2006) to simulate non-linear cosmic structures with mass and space resolutions higher than those of paper I. This is the same code that was used and tested in a related previous paper (Fullana et al. 2010), where the weak lensing effect on the CMB was studied in detail for large $\ell$ values.

As in paper I, we evolve a triply periodic model universe in a cubic simulation box. The CMB photons move in this universecrossing successive image boxes-from a suitable initial redshift to the present time. If the photons move almost parallel to the box edges, they would pass close to the same structures many times and the RS effect would undergo a large magnification due to this periodicity; however, as has been noted in many previous papers (Antón 
et al. 2005; Sáez Milán et al. 2006), it is possible to propagate photons in a periodic universe in a manner which makes negligible the aforementioned magnification. Our calculations are based on this method (ray-tracing procedure) whose main characteristics are briefly summarized below. By using this ray-tracing within the HYDRA simulations, we have been able to get small, but useful, maps of the RS temperature contrast, with an appropriate angular resolution. The analysis of these maps leads to the RS angular power spectrum for $\ell \geq 500$. Calculations of this spectrum are performed using the method described in Sáez, Holtmann \& Smoot (1996), which is based on previous work by Bond \& Efstathiou (1987). Improvements are described below.

Since the RS effect is purely gravitational, it is essentially produced by the dominant dark matter component, with contributions due to baryons are expected to be small. This suggests that $N$-body simulations may be appropriate to get a good estimate of the RS power. The suitability of this approach (without baryons) is discussed in the last paragraph of Section 5.

We assume a flat universe with scalar perturbations of an inflationary origin. The resulting inhomogeneities are then adiabatic and their statistical distribution is Gaussian. The initial conditions for our simulations are set using cosmological parameters (at the $1 \sigma$ confidence level) from the Planck collaboration as found in Planck Collaboration XVI (2014a, last column of table 5). In this reference, six parameters were adjusted to explain, at the required confidence level, current observational data from: Planck CMB temperatures (Planck), WMAP (Wilkinson Microwave Anisotropy Probe) polarization anisotropy at low $\ell \leq 23$ (WP), ACT (Atacama Cosmology Telescope) plus SPT (South Pole Telescope) anisotropy for very high $\ell$ values (HighL), and baryon acoustic oscillations (BAO). The resulting minimal fit is then named 'Planck+WP+HighL+BAO'. The values obtained for the six parameters are: (a) $\Omega_{\mathrm{b}} h^{2}=0.02214$ and $\Omega_{\mathrm{d}} h^{2}=0.1187$, where $\Omega_{\mathrm{b}}, \Omega_{\mathrm{d}}$, and $h$ are the baryon and dark matter density parameters, and the reduced Hubble constant $h=$ $10^{-2} H_{0}$, respectively, $H_{0}$ being the Hubble constant in units of $\mathrm{km}$ $\mathrm{s}^{-1} \mathrm{Mpc}^{-1}$; (b) the third value is $\theta_{\mathrm{MC}}=1.04147 \times 10^{-2}$, this parameter is the angular acoustic scale; namely, the ratio $r_{\mathrm{s}}\left(z_{*}\right) / \mathrm{d}_{\mathrm{A}}\left(z_{*}\right)$, where $r_{\mathrm{s}}\left(z_{*}\right)$ is the sound horizon at decoupling redshift $z_{*}$ and $d_{\mathrm{A}}\left(z_{*}\right)$ is the angular diameter distance for the same redshift; (c) the fourth parameter has the value $\tau=0.092$, where $\tau$ is the optical depth that characterizes reionization; and (d) the remaining two parameters are given in the formula

$P_{\mathrm{s}}(k)=A_{\mathrm{s}}\left(\frac{k}{k_{0}}\right)^{n_{\mathrm{s}}-1}$,

which gives the power spectrum of the scalar energy density perturbations at horizon crossing. The fifth and sixth parameters are $A_{\mathrm{s}}=2.20 \times 10^{-9}$ (spectrum normalization constant) and $n_{\mathrm{s}}=0.961$ (spectral index). Finally, the constant $k_{0}$, the so-called pivot scale, is taken to be $k_{0}=0.05 \mathrm{Mpc}^{-1}$ as in Planck Collaboration XVI (2014a).

The inputs required to generate the initial conditions for the HYDRA $N$-body code are calculated from the above parameters as follows: for our current purposes, energy is either dark matter $\left(\Omega_{\mathrm{d}}\right)$ or dark energy $\left(\Omega_{\Lambda}\right)$ and the universe is assumed to be flat, hence, one must use $\Omega_{\mathrm{b}}=0$ and $\Omega_{\text {matter }}=\Omega_{\mathrm{d}}=\left(1-\Omega_{\Lambda}\right)=0.307$. The values $h=$ 0.678 and $\sigma_{8}=0.826$ are also inputs for the HYDRA code: these are given in Planck Collaboration XVI (2014a) as derived parameters. Finally, the power spectrum $P(k)$ of the energy density perturbations at the initial time of the $N$-body simulation is required, and this spectrum may be calculated using the code САMB (Lewis, Challinor \& Lasenby 2000) for the Planck-adjusted parameters above.

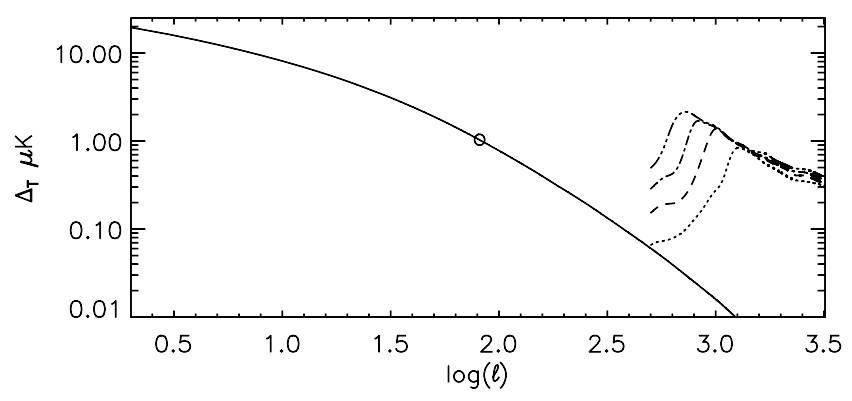

Figure 1. Quantity $\Delta_{\mathrm{T}}$ in terms of $\log (\ell)$. The solid line corresponds to the L-ISW effect. Dotted, dashed, dot-dashed, and triple-dot-dashed lines are the spectra of RS-42, RS-52, RS-62, and RS-72; namely, RS spectra for different values of $L_{\max }$ (see the text).

We choose units such that the speed of light is $c=1$ and the gravitation constant is $G=1 / 8 \pi$. For a quantity ' $A$ ', we define $A_{\mathrm{L}}$, $A_{0}$, and $A_{\mathrm{B}}$ to be the value of $A$ on the last scattering surface, at present time, and in the background, respectively; $\dot{A}\left(A^{\prime}\right)$ stands for the derivative of $A$ with respect to the conformal (cosmological) time. Symbols $\boldsymbol{x}, a, z$, and $\eta$, stand for the comoving position vector, the scale factor, the redshift, and the conformal time, respectively. We set $a_{0}=1 \mathrm{Mpc}$. The quantity $\Delta_{\mathrm{T}}=\left[\ell(\ell+1) C_{\ell} / 2 \pi\right]^{1 / 2}$, given in $\mu K$, is represented in Figs $1,3-11$ as a measure of the angular power spectrum.

This paper is organized as follows: relevant theoretical comments on the RS and the late integrated Sachs-Wolfe (L-ISW; Sachs \& Wolfe 1967) effects are presented in Section 2. Our numerical techniques: $N$-body simulations and ray-tracing are described in Section 3. Results from our simulations are presented in Section 4, where accurate RS power spectra are presented over an appropriate range of $\ell$ and, finally, in Section 5, after a summary of our results we discuss detection of the RS effect and the RS power spectrum estimation for $\ell \geq 500$.

\section{GENERAL CONSIDERATIONS ON THE L-ISW AND RS EFFECTS}

In this section, we present a brief but thorough study of the L-ISW and RS effects in the context of the $\Lambda$ cold dark matter $(\Lambda \mathrm{CDM})$ model. This study is necessary to understand our simulations as well as our discussion and conclusions.

Beginning with the L-ISW effect: this cannot be generated while matter dominates dark energy. It is generated starting a little before the end of the matter-dominated era and into the dark-energydominated period. Most of the L-ISW anisotropy is produced at low redshifts $z \leq 1$, namely, when the dark-energy density becomes of the order of the (decreasing) matter energy density. We define the L-ISW effect to be that produced by large-scale inhomogeneities evolving strictly in the linear regime; whereas a similar effect (see below) produced by weakly to strongly non-linear structures will be called the RS effect (see paper I and comments below). From these general, qualitative comments, it follows that the L-ISW study must be based on the linear approximation. We use the formalism described in Bardeen (1980) and applied in most papers discussing the CMB anisotropy (Abbott \& Schaefer 1986; Ma \& Bertschinger 1995; Seljak \& Zaldarriaga 1996; Hu \& White 1997).

We hereafter assume the longitudinal (conformal Newtonian) gauge (Ma \& Bertschinger 1995), in which, the perturbed line element has the form

$\mathrm{d} S^{2}=a^{2}\left[-(1+2 \psi) \mathrm{d} \eta^{2}+(1-2 \phi) \delta_{i j} \mathrm{~d} x^{i} d x^{j}\right]$, 
where $\psi\left(x^{i}, \eta\right)$ and $\phi\left(x^{i}, \eta\right)$ are two scalar potentials, which are identical in the absence of anisotropic stress (Bardeen 1980). Hence, in the period after decoupling $(z \leq 1100)$, the relation $\phi \simeq \psi$ is valid. Furthermore, in the same period, the universe contained a fluid with baryons and dark matter, whose total energy density and density contrast relative to the background are $\rho$ and $\delta=\left(\rho-\rho_{\mathrm{B}}\right) / \rho_{\mathrm{B}}$, respectively. There is also a constant dark-energy density $\rho_{\Lambda}$ without perturbations, which directly influences the background evolution, and indirectly, the dynamics of $\delta, \phi, \psi$, and the perturbations in general.

Let us now discuss the estimation of $\phi$ and $\psi$ and their physical meaning by starting from the gauge invariant formalism described in Bardeen (1980) where Einstein's field equations and the equations of motion are written in terms of gauge invariant variables defined in momentum space. In the longitudinal gauge, the equation 4.3 in Bardeen (1980) may be easily rewritten as follows:

$-2 \frac{k^{2}-3 K}{a^{2}} \phi=\rho_{\mathrm{B} 0}\left[\delta+3(1+W) \frac{1}{k} \frac{\dot{a}}{a} v^{(0)}\right]$,

where $K$ takes on the values $1,-1$ and 0 for a closed, open or flat background, respectively; $k$ is the wavenumber in momentum space; $W=-1$ is the ratio $P_{\mathrm{B}} / \rho_{\mathrm{B}}$; and $v^{(0)}$ is the scalar part of the fluid peculiar velocity.

For $k^{-1}(\dot{a} / a) \ll 1$, namely for physical spatial scales $k^{-1} a$ well inside the effective horizon $H^{-1}\left(k^{-1} a \ll H^{-1}\right)$, equation (3) leads to

$\phi \simeq-\left(\frac{\rho_{\mathrm{B} 0} a^{2}}{2}\right) \frac{\delta}{k^{2}}$

and, consequently, the equation satisfied by $\phi$ in position space is

$\Delta \phi \simeq \frac{1}{2} a^{2} \rho_{\mathrm{B} 0} \delta \equiv \frac{1}{2} a^{2}\left(\rho_{\mathrm{B}}-\rho_{\mathrm{B} 0}\right)$.

Hence, for subhorizon scales, $\phi$ may be interpreted as the Newtonian peculiar gravitational potential due to the matter fluid energy density perturbations $\rho_{\mathrm{B}}-\rho_{\mathrm{B} 0}$. However, for superhorizon scales, this potential does not obey equation (5).

For $K=0$ (flat background), well inside the dark-energydominated era $(W \simeq-1)$, and for all spatial scales, equation (3) may be also written in the form (4) and, consequently, equation (5) holds. Hence, we conclude that in the dark-energy period under consideration, which corresponds to redshifts smaller than unity, the potentials $\psi$ and $\phi$ are identical and, moreover, they are Newtonian gravitational potentials for any scale $k$ (either linear or non-linear).

For perturbations evolving in the linear regime, the equations of the null geodesics corresponding to the line element (2) may be used to prove that the $\mathrm{CMB}$ undergoes the following integrated effect:

$\frac{\Delta T}{T_{\mathrm{B}}}(\boldsymbol{n})=2 \int_{\eta_{\mathrm{L}}}^{\eta_{0}} \frac{\partial \phi(\boldsymbol{x}, \eta)}{\partial \eta} \mathrm{d} \eta$,

where $\Delta T / T_{\mathrm{B}}=\left(T-T_{B}\right) / T_{\mathrm{B}}$ is the temperature contrast with respect to $T_{\mathrm{B}}$ (the averaged CMB temperature). A good approximation to $\Delta T / T_{\mathrm{B}}$ may be obtained by calculating the above integral along the background null geodesic corresponding to the unit line-of-sight vector $\boldsymbol{n}$. This is the so-called Born approximation, which has been shown to be adequate in the $\ell$ interval $300 \leq \ell \leq 5000$, where we estimate the RS power (see Van Waerbeke et al. 2001; Hilbert et al. 2009).

A vanishing anisotropic stress and the condition $\phi \simeq \psi$ may be assumed to estimate the L-ISW effect and, then, the integrand of equation (6) may be easily calculated (in momentum space) from equations 18 and 21.d of Ma \& Bertschinger (1995). The result is

$\dot{\phi}(k, \eta)=\dot{\eta}-\frac{\dot{a}}{a} \eta-\frac{1}{2 k^{2}}\left[\frac{\ddot{a}}{a}-3\left(\frac{\dot{a}}{a}\right)^{2}\right](\dot{h}+6 \dot{\eta})$,

where, $h(k, \eta)$ and $\eta(k, \eta)$ are scalar modes associated with the metric in the synchronous gauge (see Ma \& Bertschinger 1995; Bardeen 1980). Since CMBFAST (Seljak \& Zaldarriaga 1996) and CAMB use this gauge, these codes calculate all the functions involved in the right-hand side of equation (7) and, consequently, $\dot{\phi}(k, \eta)$ and the angular power spectrum of the L-ISW may be easily obtained. Both codes lead to very similar spectra. The solid line of Fig. (1) represents the spectrum obtained by using CMBFAST. The calculation of the L-ISW spectrum performed here is more accurate than the estimate presented in paper I, which is based on the approximating assumption that, while the L-ISW effect is produced, any spatial scale evolves according to the $\delta$ growing mode given by Peebles (1980). This is valid for subhorizon scales, but superhorizon scales only evolve in the same way for $W=-1$, which is not strictly valid while the L-ISW effect is being generated: namely, while matter and dark energy have comparable energy density and $W$ is only close to, but not strictly equal to -1 . As soon as slightly nonlinear scales-say, scales with density contrasts greater than about 0.2 - contribute to a given multipole $\ell$, an RS effect is produced and $C_{\ell}$ deviates from the value given in the solid line of Fig. 1. Appropriate simulations may be used to estimate the point at which this deviation starts (see Section 5). In Cai et al. (2010, top-left panel of fig. 17), it is shown that, if simulations of the L-ISW-RS effects start at redshift $z \simeq 10$, this point corresponds to $\ell \sim 80$, marked by the small circle in Fig. 1 . As it is verified below, the scales producing effects close to $\ell \sim 80$ are not strongly non-linear and, consequently, suitable approximations seem to be useful to estimate the RS effect produced by them. We are not concerned here with these scales and approximations, which would allow us to link the solid line (strictly linear structures) and a line giving the total RS power in the region of the discontinuous lines, which would be a certain limit of these lines (see Section 5). This link would lead to a unique spectrum valid for any scale; nevertheless, we are mostly interested in the form of the spectrum in the $\ell$ interval of the discontinuous lines, which describes the RS effect produced by strongly and mildly non-linear structures, whose computation properly requires $N$-body simulations.

Let us now consider the RS effect, whose definition and estimation are based on the reasonable assumption that, even for non-linear cosmological structures such as clusters, superclusters, and voids, the line element maintains the form (2) with $\phi \simeq \psi \ll 1$, as holds just before the perturbations leave the linear regime while they are evolving well inside the effective horizon (see above). This assumption has not been rigorously proved: e.g. in the non-linear regime, the line element might involve two different small potentials $(\phi \neq$ $\psi)$; in other words, deviations from the line element (2) are possible in spite of the fact that, at the end of the linear regime, we have proved that the condition $\phi \simeq \psi$ is satisfied, and also that $\phi$ and $\psi$ are Newtonian gravitational potentials satisfying equation (5). Once the aforementioned assumption is accepted, it may be easily proved that equation (6) is also valid to calculate the CMB integrated effect due to non-linear structures, that is, the RS effect.

For $\ell>10^{3}$, the RS effect is essentially produced by dark matter inhomogeneities evolving in the mildly and strongly non-linear regimes, thus well inside the effective horizon. A box with a size of a few hundred megaparsecs would contain a statistically significant distribution of structures with spatial scales smaller than a few tens 
of megaparsecs such as clusters, superclusters, voids, and so on. Within a simulation box of this scale, the total velocities of the dark matter particles (Hubble plus peculiar component) are much smaller than unity, gravitation is weak, and equation (5) holds; hence, Newtonian $\mathrm{N}$-body simulations are adequate to allow us to estimate the RS effect.

\section{NUMERICAL TECHNIQUES}

Following from the comments of Sections 1 and 2, we adopt the following numerical approach: an $\mathrm{N}$-body code to simulate structure formation in sufficiently large regions; a ray-tracing procedure to move photons in the simulated periodic universe avoiding spurious RS magnification; and a method to extract the angular power spectrum from small maps of RS temperature contrasts. For completeness, in the following sections, we present a brief description of these three techniques.

\section{1 $N$-body simulations}

The simulations presented hereafter were run using a parallel OpenMP-based implementation of the 'HYDRA' code (Thacker \& Couchman 2006). This code uses the AP3M algorithm to compute gravitational forces within a simulation containing $N_{\mathrm{p}}$ particles. In the AP3M algorithm, a cubic 'base' mesh of size $N_{\mathrm{c}}$ cells per side is supplemented by a series of refined-mesh P3M calculations to provide submesh resolution. Gravitational softening is implemented using the S2 softening kernel (Hockney \& Eastwood 1988) which is remarkably similar in shape to the cubic spline softening kernel used in many treecodes (e.g. Hernquist \& Katz 1989). The S2 softening used in the kernel is approximately $2.34 \times S_{\mathrm{p}}$ where $S_{\mathrm{p}}$ is an equivalent Plummer softening length, which we quote throughout the paper to enable a simple comparison to other work. The softening length is held constant in physical coordinates subject to the resolution not falling below 0.6 of the mean interparticle spacing. This technique is widely applied (e.g. Springel et al. 2005) and is a compromise between assuring that the potential energy of clusters does not evolve significantly at low redshift, while still ensuring that structures and linear perturbations at high redshift are followed with reasonable accuracy.

Initial conditions were calculated using the standard Zel'dovich approximation technique (Efstathiou et al. 1985), and all simulations were started at a redshift of $z=50$, which is sufficiently early to place modes in the linear regime.

The free parameters characterizing our $\mathrm{N}$-body simulations are the box size, $L_{\text {box }}$, the number of dark matter particles, $N_{\mathrm{p}}$, the number of cells per edge, $N_{\mathrm{c}}$, and the softening parameter $S_{\mathrm{p}}$.

\subsection{Ray-tracing}

Several methods have been developed to calculate the CMB anisotropies produced by the peculiar gravitational potential of non-linear structures. Most of them are based on simulations of non-linear structure evolution in finite boxes. CMB photons move through these boxes which cover the photon world lines. Specifically, photons are located at time, $t$, inside one simulation box, whose structures have been evolved to this time $t$. There is systematic noise (magnification) associated with the motion of CMB photons in a periodic universe covered (at any time) by identical boxes. Nevertheless, this magnification strongly depends on the specifics of the ray-tracing scheme, namely, on the photon paths,

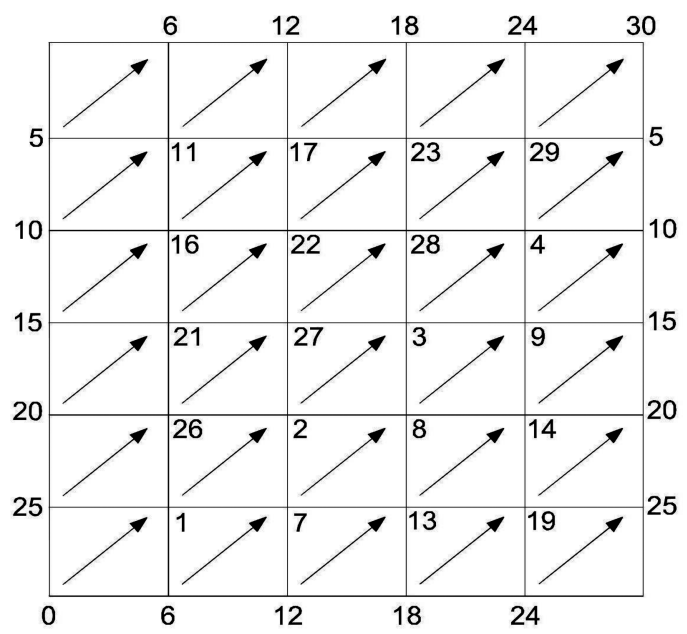

Figure 2. Sketch of the photon motion along a certain preferred direction. The big square is a generic $(x, y)$ plane separating boxes. Photons travel through successive boxes numbered from 0 to 29 . The crossing pointlabelled $i$-from the $i-1$ to the $i$ box belongs to the common $(x, y)$ plane separating these boxes. Points on the square edges are identified due to box periodicity.

which must be chosen to avoid repeatedly sampling the same structures in the periodic images. Various methods have been designed to achieve this goal, see, e.g. Tuluie, Laguna \& Anninos (1996), Jain, Seljak \& White (2000), and Cai et al. (2010).

Other approaches use random translations and orientations of the simulation boxes, which lead to discontinuities at the points where the CMB photons cross from one box to its successor (Springel, White \& Hernquist 2001). An improvement on this approach was designed by Carbone et al. (2008) to minimize the possible effect of discontinuities; while the signal from the residual discontinuities is likely small, our ray-tracing has the advantage of avoiding it completely.

There are also tiling methods, which are based on independent $\mathrm{N}$-body simulations with decreasing sizes that telescope in resolution along the line of sight (Hamana, Colombi \& Suto 2000; White $\& \mathrm{Hu}$ 2001; Sato et al. 2009; Barreira et al. 2016). Since the simulations are different, there are also discontinuities in crossing from box to box.

Our ray-tracing approach is optimized from a number of perspectives. Identical comoving boxes cover the universe at any time. The periodicity of the boxes guaranties that there are no discontinuities in the matter field anywhere. Photons move through the simulation volume along specially chosen directions to avoid 'periodicity magnification'. The existence of these directions was first pointed out in Antón et al. (2005). In Sáez Milán et al. (2006) and also in paper I, our ray-tracing was improved by introducing a cutoff, which avoids the power on all spatial scales greater than a certain limit, $L_{\max }$, in the calculation of the integrand in equation (6).

The sketch of Fig. 2 shows how photons move along a particular preferred direction, $P_{1}$. Axes $x, y$, and $z$ are parallel to the edges of the simulation boxes. Angles $\theta$ and $\varphi$ are spherical coordinates defined with respect to these axes. Photons leaving, or arriving at, the observer along $P_{1}$ will cross successive $(x, y)$ faces. In the sketch, point 0 is the observer position and point $i$ represents photon locations on the $i$ th face at crossing time. All these points are represented on the same generic $(x, y)$ plane, although they may belong to different boxes. Inside the first box, a photon moves from point 0 to point 1 and it enters then into the second box. If the angles defining 
the $P_{1}$ direction-in radians - are chosen to be $\theta=0.2547$ and $\varphi=0.8771$, and the comoving box size is $L_{\mathrm{box}}=512 \mathrm{~h}^{-1} \mathrm{Mpc}$, the distance from points 0 to 1 -in the $(x, y)$ plane-is found to be $D=1332949 h^{-1} \mathrm{Mpc}$, this implies that photons enter two successive boxes at points $i$ and $i+1$ separated by distance $D$, which is much greater than any assumed value of $L_{\max }$ and, then, on account of the fact that the power on scales $L>L_{\max }$ has been avoided, photons moving along $P_{1}$ enter successive boxes through independent uncorrelated regions (i.e. there are negligible periodicity effects). In Fig. 2, we see that a photon may cross 30 successive boxes before arriving at the box zone in which the observer is located. Since the comoving distance from $z=25$ to 0 is $\sim 7700 h^{-1} \mathrm{Mpc}$ and the distance travelled inside any box is $526.6361 h^{-1} M p c$, photons coming from $z=25$ only travel through 15 successive boxes before reaching the observer. As explained in detail in Sáez Milán et al. (2006), there is no unique preferred direction. Various preferred directions were considered in Fullana et al. (2010), where it was shown that, for $\mathrm{CMB}$ weak lensing, different directions lead to essentially equivalent results.

The parameters involved in the ray-tracing procedure are the following: a number of directions, $N_{\text {dir }}$, per edge of the square CMB map (one per pixel, $N_{\text {dir }}=N_{\text {pix }}$ ); an initial redshift, $z_{\text {in }}$, for the RS calculation; a photon step, $\Delta_{\mathrm{ps}}$, used to perform the integral in equation (6); the length $L_{\max }$ defining the cutoff; and angles $\theta$ and $\varphi$ defining the preferred direction.

Hereafter, an RS simulation (RSS) is the calculation of the RS integral along the pixel directions, plus the construction of the maps and the estimation of $C_{\ell}$ multipoles (angular power spectra). An RSS is characterized by the parameters and initial conditions required by the $N$-body simulation together with the parameters of the ray-tracing procedure. We define reference RSSs using the following parameters: $L_{\text {box }}=512 f h^{-1} \mathrm{Mpc}$, where $f=1.35$ (see below), $N_{\mathrm{p}}=256^{3}, N_{\mathrm{c}}=1024, S_{\mathrm{p}}=50 \mathrm{~h}^{-1} \mathrm{kpc}, N_{\text {dir }}=256, L_{\max }=42 h^{-1}$ $\mathrm{Mpc}, z_{\text {in }}=25$, and $\Delta_{\mathrm{ps}}=125 \mathrm{~h}^{-1} \mathrm{kpc}$. The resolution of these simulations is $\sim 5 S_{\mathrm{p}}=250 \mathrm{~h}^{-1} \mathrm{kpc}$. The angular size, $\Phi_{\text {map }}$, of the resulting square RS maps is $3.6 f \simeq 4.9$, and their angular resolution is $\Delta_{\text {ang }} \simeq f 0.85 \operatorname{arcmin} \simeq 1.15 \operatorname{arcmin}\left(\ell \simeq 12700 f^{-1} \simeq 9400\right)$. There are infinite possible realizations of this type of RSS corresponding to different initial conditions for the $N$-body simulation as well as to distinct preferred directions. In Section 4, we consider the effect of parameter changes and demonstrate that the calculation of the power spectra ( $C_{\ell}$ multipoles) is robust and accurate at least for $500 \leq \ell \leq 3200$.

\subsection{Calculation of the RS integral}

Given a line of sight with unit vector $\boldsymbol{n}$, a numerical integration must be performed to estimate the RS temperature contrast predicted by equation (6). Integration is done along the background null geodesic corresponding to $\boldsymbol{n}$ (this is the Born approximation, as noted above). The integration (photon) step is assumed to be constant, $\Delta_{\mathrm{ps}}$. This step defines a set of regularly spaced integration points on the background null geodesic, and the integrand, $\partial \phi(\boldsymbol{x}, \eta) / \partial \eta$, must be evaluated at each of these points. We have verified in previous work (e.g. Fullana et al. 2010) that, (conservatively) assuming a Plummer softening of $S_{\mathrm{p}}$ gives an effective resolution of $5 S_{\mathrm{p}}$ (Moore et al. 1998), our simulations exhibit converged results with 2.5 photon steps per effective resolution element. Moreover, we have shown that this choice ensures convergence of the calculation of lensed properties up to very high $\ell\left(>10^{4}\right)$.

The $N$-body simulations self-consistently treat all spatial scales within the box, including scales larger than the cutoff, $L_{\max }$. The cutoff is only used to calculate the quantity $\partial \phi(\boldsymbol{x}, \eta) / \partial \eta$ in the integral. In this way, structure formation is not affected by the cutoff, but scales $L>L_{\max }$ do not contribute to the RS temperature contrast. The same type of cutoff was used in Fullana et al. (2010) to calculate weak lensing deviations.

Since equation (6) gives the total integrated effect due to linear and non-linear structures, the limits of the integral involved in this equation are $\eta_{\mathrm{L}}$ and $\eta_{0}$. However, the L-ISW effect produced by the linear structures has been accurately estimated (see Fig. 1) and, consequently, only the time interval in which there are non-linear structures having relevant contributions to the integrated effect must be included. This interval will be appropriately established in Section 4; for now, it is sufficient to say that the limits of this interval will be $\eta_{\text {in }}$ and $\eta_{0}$ with $\eta_{\text {in }} \ll \eta_{\mathrm{L}}$. The integral in equation (6) will be extended to this interval, which corresponds to the redshift interval $\left(z_{\text {in }}, 0\right)$. We have also calculated the integral in intervals $\left(z_{\text {in }}, z_{\mathrm{f}}\right)$ for various small values $\left(z_{\mathrm{f}}>0\right)$ in order to estimate the RS contribution from low redshifts (see Section 4.5).

Finally, our algorithm for determining $\partial \phi(x, \eta) / \partial \eta$ at the integration points of a given background null geodesic is as follows:

(i) use the photon step, $\Delta_{\mathrm{ps}}$, to determine all integration points (radial coordinate and time) for $\eta>\eta_{\text {in }}$;

(ii) place a test particle (which does not influence the $N$-body simulation) at each integration point;

(iii) calculate the potential $\phi$ for the test particles just as is done in the $N$-body code: namely, using the long-range fast Fourier transform (FFT) component and short-range PP correction as in the HYDRA algorithm. However, during the FFT convolution for the test particles eliminates contributions from scales larger than $L_{\max }$ by removing the signal from wavenumbers $k<2 \pi / L_{\max }$;

(iv) given the time, $\eta$, for each test particle find the time steps, $i$ and $i+1$, of the $N$-body simulation such that $\eta_{i}<\eta<\eta_{i+1}$ and use $\phi\left(\eta_{i}\right)$ and $\phi\left(\eta_{i+1}\right)$ to calculate the derivative $\partial \phi(\boldsymbol{x}, \eta) / \partial \eta$ at the position of the chosen test particle.

Our method does not smooth on to grids or create RS planes 'on the fly'. Nor is simulation data stored for CMB post-processing. In order to build up our maps of the RS effect, the integrals are calculated along many null geodesics, and calculations are performed simultaneously over all these geodesics as the code runs. As a consequence of not storing the data, if the parameters associated with the ray-tracing (see Section 3.2) are varied, a new $N$-body simulation is necessary. The ray-tracing used here was already implemented (Fullana et al. 2010) in a Lagrangian code (HYDRA), and another ray-tracing on the fly without data storing has been recently implemented (Barreira et al. 2016) in a Eulerian $N$-body code (RAMSES). In both cases, the distribution of particles sets what is smoothed. What matters is the effective resolution of the rays in angle and in space. As long as either grids, or the approach taken in our paper, are both beyond a certain limit of resolution the results should be similar (modulo different assumptions about smoothing kernels and so on). So far we have pointed out resemblances, let us now list some differences, e.g. our ray-tracing applies to Lagrangian $N$-body codes, it uses only a simulation in each run, discontinuities from box to box are prevented, and a cutoff on spatial scales (very useful for scale analysis, see below) is performed to make periodicity magnification negligible; however, the ray-tracing of Barreira et al. (2016) applies to Eulerian codes, various simulations are necessary in every run to prevent magnification, and there are discontinuities from box to box. 


\subsection{RS small maps: construction and analysis}

To build maps of the RS temperature contrast, we proceed as follows: a preferred direction is selected and it is assumed that this direction points towards the centre of a square map, which is uniformly pixelized by choosing a certain number of pixels, $N_{\text {pix }}$, per edge. There is an observation direction pointing towards the centre of each pixel. The angular size of these maps may be easily calculated by taking into account that all the observation directions (map pixels) are inside the solid angle subtended by a face of the simulation box (orthogonal to the central line of sight) at redshift $z_{\text {in }}$. The angular resolution is then $\Delta_{\text {ang }}=\Phi_{\text {map }} / N_{\text {pix }}$, where $\Phi$ is the angle subtended by the side of the map. As a useful example, for $z_{\text {in }}=6$, which was the initial redshift in all the simulations considered by us in Fullana et al. (2010), a box size $L_{\text {box }}=512 h^{-1}$ Mpc leads to a square sky patch with angular size $\Phi_{\text {map }}=4.96$, whose angular resolution, for $N_{\text {pix }}=256$, is $\Delta_{\text {ang }} \sim 1.16$ arcmin $\left(\ell=10800 / \Delta_{\text {ang }} \simeq 9300\right)$. If the value $N_{\text {pix }}=256$ is fixed, as in this paper, then $\Phi_{\text {map }}\left(\Delta_{\text {ang }}\right)$ is proportional to $L_{\text {box }}\left(1 / L_{\text {box }}\right)$.

Since the angular size of all the maps considered in this paper is small (see below), the directions of all the pixels may be considered as preferred ones and, consequently, RS integrals can be calculated for each pixel across the full map. Moreover, maps cover small parts of the celestial sphere and may be regularly pixelized as flat. In this situation, the power spectrum estimator described in Sáez et al. (1996) applies. Since this estimator uses the FFT, it has been improved by assuming mirror symmetric boundary conditions (Pratt 2013).

For $z_{\text {in }}>6$, e.g. $z_{\text {in }}=25$ (initial redshift of the reference RSSs), boxes with $L_{\text {box }}=512 h^{-1} M p c$ lead to maps having $\Phi_{\text {map }} \sim 3.6$. Since these maps seem to be a little small, we have chosen a larger box with $L_{\text {box }}=f 512 h^{-1} \mathrm{Mpc}$ and $f>1$, which leads to a more extended map with $\Phi_{\text {map }} \sim(3.6 f)$. The $f$ value must be suitably chosen for every $z_{\text {in }}>6$. Larger boxes (maps) correspond to lower resolutions (angular resolutions) in the $N$-body (RS) simulation; hence, $f$ cannot be too large. The criterion used, in Section 4.6, to fix the $f$ value for each $z_{\text {in }}$ is as follows. The square with $L_{\text {box }}=$ $f 512 \mathrm{~h}^{-1} \mathrm{Mpc}$, orthogonal to the central line of sight, subtends a solid angle at a given $z_{\text {in }}(>6)$; the $f$ value is chosen such that the square having $L_{\text {box }}=512 h^{-1} \mathrm{Mpc}$, placed at redshift $z=6$, subtends the same solid angle. This choice gives $f$ values producing admissible reductions of resolution (see below) and more extended $(3.6 f) \times(3.6 f) \mathrm{RS}$ maps. As noted in Section 3.2, for $z_{\text {in }}=25$, one obtains $f=1.35$.

\section{RESULTS}

We next analyse the effect of varying key $N$-body and ray-tracing parameters. The reference RSSs defined above play a central role in this analysis. The parameters of the reference simulations are varied either singly or in constrained pairs as reported in the following sections. In this way, we estimate the accuracy of the resulting RS angular power spectrum and highlight its main properties.

\subsection{On the number of reference RSSs leading to accurate $C_{\ell}$ multipoles}

Since our RS maps are small statistical samples, different initial conditions do not give exactly the same $C_{\ell}$ coefficients. The RS angular power spectrum is obtained as the average of a number of simulations corresponding to distinct initial modes. We have investigated how many reference RSSs are needed to get a sufficiently

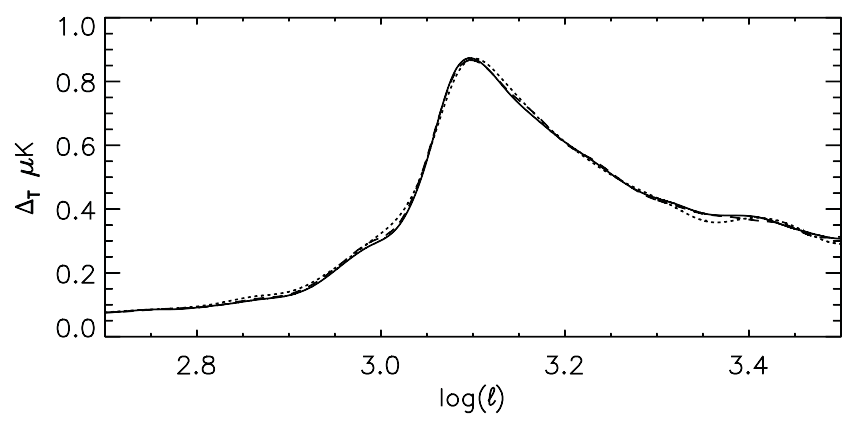

Figure 3. Quantity $\Delta_{T}$ as a function of $\log (\ell)$ for varying numbers of averaged RSSs. The averaged power $\Delta_{\mathrm{T}}$ obtained with three (dotted line), six (dashed), and nine (solid) reference RSSs are shown.

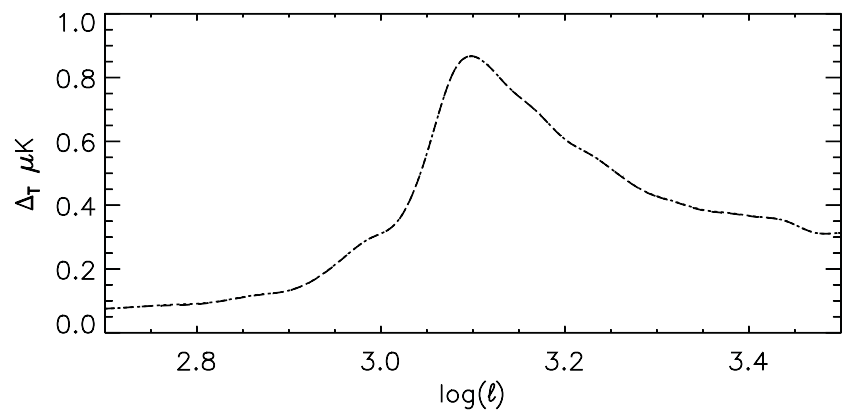

Figure 4. $\Delta_{\mathrm{T}}$ power spectrum in terms of $\log (\ell)$ for different final RS integration/simulation redshifts. Dotted (dashed) line corresponds to a reference RSS which has been stopped at $z_{\mathrm{f}}=0.5\left(z_{\mathrm{f}}=0.2\right)$. The curves are indistinguishable.

accurate, converged spectrum. In Fig. 3, we show the averaged power spectra obtained by using 3, 6, and 9 runs. As can be seen, very similar results have been found for the three cases. Hereafter, we give spectra found by averaging six independent simulations. Puchades et al. (2006) found that about 30 PM simulations were necessary for a converged spectrum. The smaller number of simulations required here is due to the higher resolution of our HYDRA simulations.

\subsection{Effect of changing the final ray-tracing redshift $z_{\mathrm{f}}$}

Spectra corresponding to the $z_{\mathrm{f}}$ values of 0.5 and 0.2 are shown in Fig. 4. Evidently, these two spectra are almost indistinguishable, indicating that the signal produced between redshifts 0.5 and 0.2 contributes negligibly to the total RS effect. We have also verified that the contribution from 0.2 to 0 is negligible as expected. The same result was found in the PM simulations of Puchades et al. (2006).

We also considered other variations from the reference RSSs such as different boxes, particle numbers and so on. The conclusion is the same in all the cases: namely, that there is a negligible contribution between $z=0.5$ and 0 . Despite this, we evolve all the simulations to redshift $z_{\mathrm{f}}=0.2$ (rather than to $z_{\mathrm{f}}=0.5$ ). The simulations are stopped at this redshift to reduce the CPU time, since the $N$-body code progresses very slowly from $z=0.2$ to 0 , particularly for boxes with many particles.

The fact that the RS effect becomes negligible at $z<0.5$ is not surprising since, at these small redshifts in the modelled cosmology, non-linear structures such as voids, clusters, and superclusters become essentially stable and, consequently, produce very small values of the time derivative $\partial \phi(x, \eta) / \partial \eta$ in equation (6). In the 


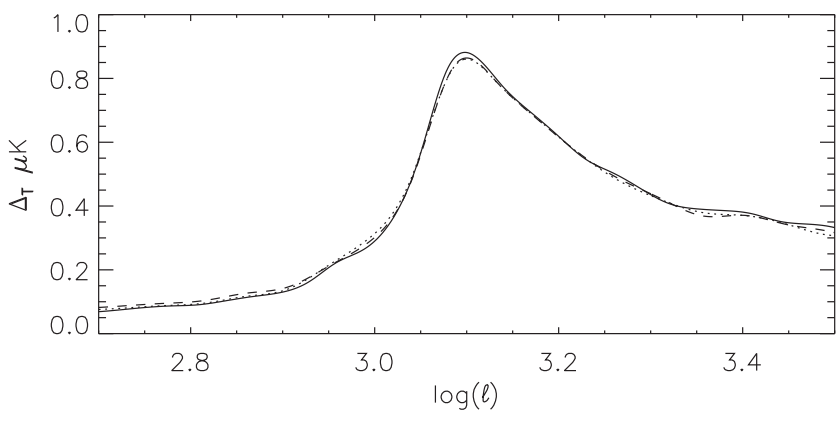

Figure 5. Quantity $\Delta_{\mathrm{T}}$ as a function of $\log (\ell)$ for varying particle smoothing and photon step. Solid, dotted, and dashed curves correspond to $S_{\mathrm{p}}=75 h^{-1} \mathrm{kpc}, S_{\mathrm{p}}=50 h^{-1} \mathrm{kpc}$, and $S_{\mathrm{p}}=25 h^{-1} \mathrm{kpc}$, respectively. The photon step is $\Delta_{\mathrm{ps}}=2.5 S_{\mathrm{p}}$ in each case. All other parameters are the same as for the reference simulations.

case of weak lensing, which is produced by the transverse gradient of $\phi$, it has been showed that, for $\ell>350$, the signal produced at $z<0.2$ is also negligible (Carbone et al. 2008; Fullana et al. 2010).

\subsection{Impact of spatial resolution}

We now consider the effect of varying the parameters $S_{\mathrm{p}}$ (smoothing) and $\Delta_{\mathrm{ps}}$ (photon step) on the RS angular power spectra. For a given $S_{\mathrm{p}}$, a photon step satisfying the condition $S_{\mathrm{p}} / \Delta_{\mathrm{ps}} \simeq 0.5$ has been found to be good enough to take advantage of the effective $N$-body resolution in the integrations involved in any RSS; this condition leads to 2.5 photon steps per effective resolution interval. The same condition is suitable for weak lensing (see Section 3.3).

In this section, we consider three values of $S_{\mathrm{p}}$ : one is the reference RSSs value, $S_{\mathrm{p}}=50 h^{-1} \mathrm{kpc}$; the other two are $S_{\mathrm{p}}=25 h^{-1} \mathrm{kpc}$ and $S_{\mathrm{p}}=75 h^{-1} \mathrm{kpc}$. The corresponding values of $\Delta_{\mathrm{ps}}$ are calculated using the above condition $\Delta_{\mathrm{ps}}=2 S_{\mathrm{p}}$. The remaining parameters of these RSSs are unchanged from the reference (see Section 3.2). The angular power spectra obtained in these simulations are shown in Fig. 5, where it can be seen that the three spectra are very similar. All values of $S_{\mathrm{p}}$ lead to almost identical $C_{\ell}$ coefficients for $500 \lesssim \ell \lesssim$ 3200. To quantify the separation between the solid $\left(S_{\mathrm{p}}=75 h^{-1}\right.$ $\mathrm{kpc})$ and the dashed $\left(S_{\mathrm{p}}=25 h^{-1} \mathrm{kpc}\right)$ lines, we have calculated the variance $\sigma_{1}^{2}=M^{-1} \sum_{k=1}^{M}\left[\Delta_{\mathrm{T}}(\text { solid })-\Delta_{\mathrm{T}}(\text { dashed })\right]^{2}$, where $M$ is the number of $\Delta_{\mathrm{T}}$ values numerically calculated in the $\log (\ell)$ interval $(2.7,3.5)$. The result is $\sigma_{1}^{2} \simeq 1.22 \times 10^{-4}$ (very close spectra). In this and the next sections, we calculate variances for other pairs of spectra in appropriate $\log (\ell)$ intervals, these variances are numbered with the subscript $i$ in the form $\sigma_{i}^{2}$, and they allow us quantitative comparisons of spectra separations.

We have also compared the CMB spectra obtained from two sets of simulations having different box sizes, but the same value of $L_{\text {box }} / f N_{\mathrm{p}}^{1 / 3}$. In these simulations (shown in Fig. 6), all parameters except $L_{\mathrm{box}} / f$ and $N_{\mathrm{p}}$ have the same values as the reference RSSs.

In the first comparison (top panel), one simulation has $L_{\text {box }} / f=$ $1024 h^{-1} \mathrm{Mpc}$ and $N_{\mathrm{p}}=256^{3}$ (dashed line), and the other $L_{\text {box }} / f=$ $512 h^{-1} \mathrm{Mpc}$ and $N_{\mathrm{p}}=128^{3}$ (solid line). The factor $f$ is the same for both simulations $(f=1.35)$ and the simulations thus have the same density of particles $N_{\mathrm{p}} / L_{\text {box }}^{3}$ or physical particle mass. We see that the resulting spectra are similar. The variance corresponding to the two curves of this panel, in the interval $(2.7,3.5)$, is $\sigma_{2}^{2} \simeq$ $1.87 \times 10^{-3}>\sigma_{1}^{2}$.

In the second set of simulations (bottom panel), the ratio $L_{\text {box }} / f N_{\mathrm{p}}^{1 / 3}$ is $1 / 8$ that of the first set corresponding to eight times better mass resolution. Three different box sizes are compared (see
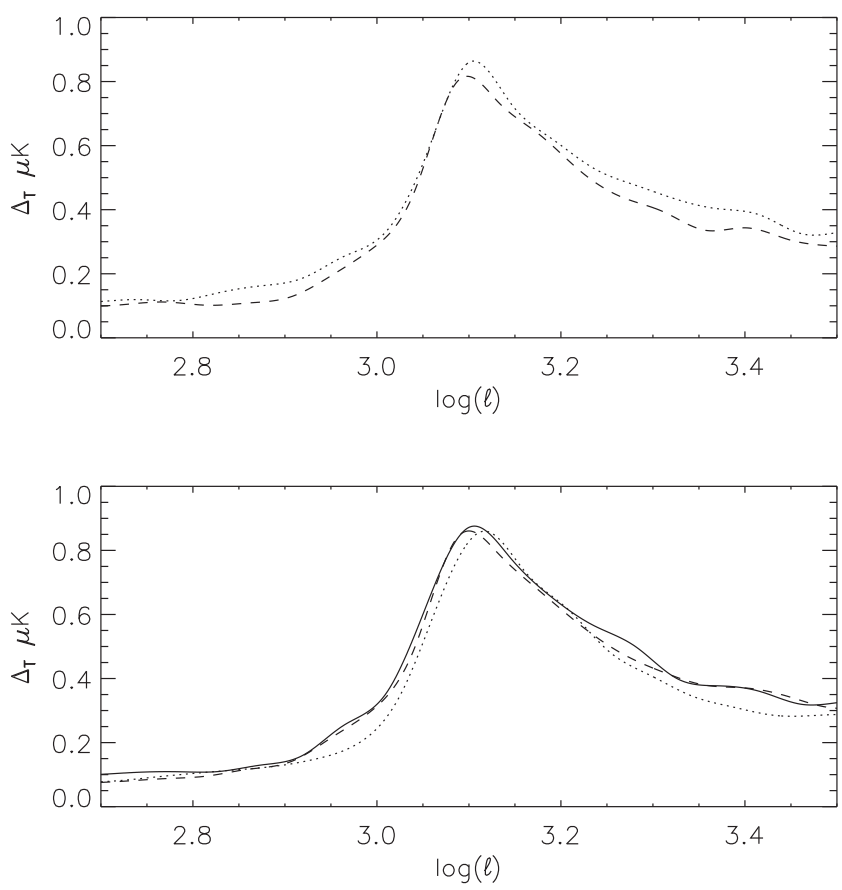

Figure 6. Quantity $\Delta_{\mathrm{T}}$ as a function of $\log (\ell)$ for varying box size at constant particle mass. Top panel: dotted (dashed) line corresponds to a box size of $1024 f h^{-1} \mathrm{Mpc}\left(512 f h^{-1} \mathrm{Mpc}\right)$ and $N_{\mathrm{p}}=256^{3}\left(N_{\mathrm{p}}=128^{3}\right)$ particles. The remaining parameters take on the same values as in the reference RSSs. Bottom panel: as in the top panel for $L_{\text {box }}=1024 f h^{-1} \mathrm{Mpc}$ and $N_{\mathrm{p}}=$ $512^{3}$ (solid line); $L_{\text {box }}=512 f h^{-1} \mathrm{Mpc}$ and $N_{\mathrm{p}}=256^{3}$ (dashed; reference RSS); and $L_{\text {box }}=256 f h^{-1} \mathrm{Mpc}$ and $N_{\mathrm{p}}=128^{3}$ (dotted).

figure caption for details). Again, it can be seen that the computed RS spectra are rather stable to these parameter variations, but the dashed and solid lines are very close and the dotted curve separation from both is appreciable, which indicates convergence towards a spectrum close to that of the reference RSSs (dashed line in the bottom panel). Accordingly, in the interval $(2.7,3.5)$, the variance associated with the continuous and dotted (continuous and dashed) lines is $\sigma_{3}^{2} \simeq 2.42 \times 10^{-3}\left(\sigma_{4}^{2} \simeq 3.62 \times 10^{-4}\right)$. Quantity $\sigma_{3}^{2}$ is of the same order as $\sigma_{2}^{2}$, but $\sigma_{4}^{2}$ is much smaller (convergence).

\subsection{Impact of mass resolution}

In this section, we change the mass resolution by varying the number of particles, $N_{\mathrm{p}}$, in a fixed volume. The chosen values of $N_{\mathrm{p}}$ are $128^{3}, 256^{3}$, and $512^{3}$; the box size, $L_{\text {box }}=512 f h^{-1} \mathrm{Mpc}$, and all the remaining parameters are identical to those of the reference RSSs. The particle masses are $7.0 \times 10^{12} \mathrm{M}_{\odot}$ for $N_{\mathrm{p}}=128^{3}$; $8.4 \times 10^{11} \mathrm{M}_{\odot}$ for $N_{\mathrm{p}}=256^{3}$ (reference RSS); and $1.1 \times 10^{11} \mathrm{M}_{\odot}$ for $N_{\mathrm{p}}=512^{3}$. The resulting angular power spectra are shown in Fig. 7, where we see that there is a smooth dependence on mass resolution; in fact, in the interval $(2.7,3.5)$, the variance corresponding to the solid $\left(N_{\mathrm{p}}=512^{3}\right)$ and dashed $\left(N_{\mathrm{p}}=128^{3}\right)$ lines is $\sigma_{5}^{2} \simeq 2.21 \times 10^{-3}$; which has the same order as $\sigma_{2}^{2}$ and $\sigma_{3}^{2}$ (spatial resolution in Section 4.3). The dependence on mass resolution is much greater than the dependence on $S_{\mathrm{p}}$ variations studied in Section $4.3\left(\sigma_{1}^{2} \simeq 1.22 \times 10^{-4}\right)$. For $\ell \gtrsim 1200$, the power of the $\mathrm{RS}$ signal is a generally slowly increasing function of $N_{\mathrm{p}}$, and convergence towards a spectrum very close to that of $N_{\mathrm{p}}=512^{3}$ is evident. The spectrum corresponding to $N_{\mathrm{p}}=256^{3}$ is very similar to that of $N_{\mathrm{p}}=512^{3}$ and, consequently, the spectrum obtained with the 


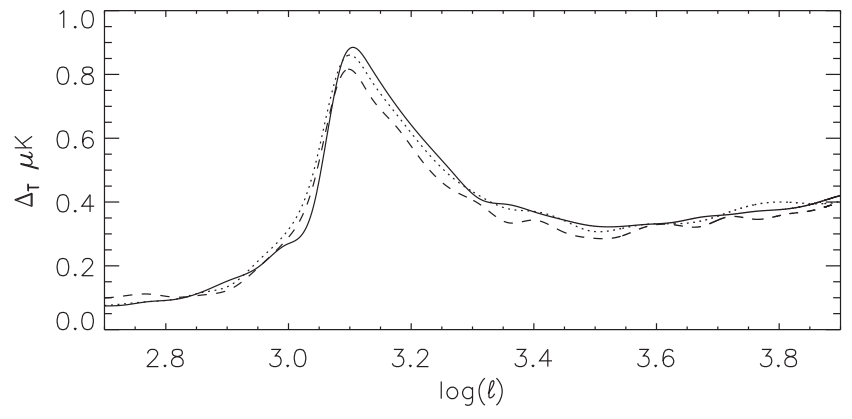

Figure 7. $\Delta_{\mathrm{T}}$ power spectrum in terms of $\log (\ell)$ for varying mass resolution. All simulation parameters are the same as for the reference RSSs, except $N_{\mathrm{p}}$, which takes the values $128^{3}, 256^{3}$, and $512^{3}$, for the dashed, dotted, and solid lines, respectively.

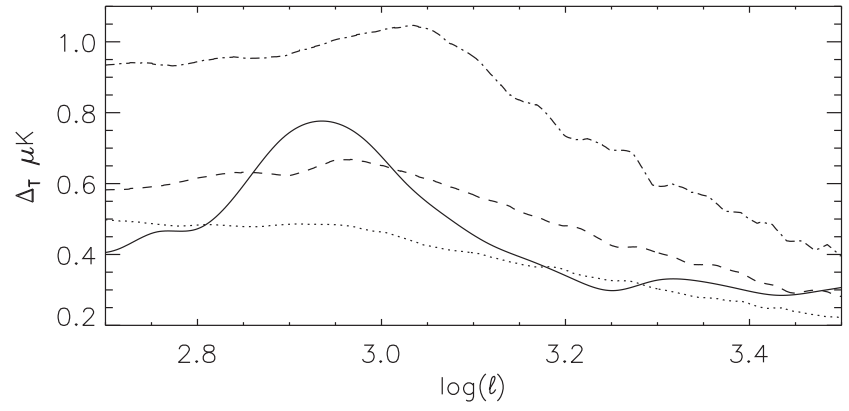

Figure 8. Quantity $\Delta_{\mathrm{T}}$ as a function of $\log (\ell)$. Dotted, dashed, and dotdashed lines correspond to PM simulations (Fig. 6 of paper I) with effective resolutions of $0.5,1$, and $2 \mathrm{Mpc}$, respectively. Solid line is an AP3M HYDRA simulation, with an effective resolution $\sim 0.37 \mathrm{Mpc}$.

reference RSSs may be considered a very good approximation to the RS angular power spectrum. A quantitative confirmation of this convergence is obtained by estimating the variance of the dotted and solid lines in the interval $(3.1,3.7)$, whose small value is found to be $\sigma_{6}^{2} \simeq 3.11 \times 10^{-4}$. In the interval $(2.7,3.5)$, we have found the variance $\sigma_{7}^{2} \simeq 7.68 \times 10^{-4}>\sigma_{6}^{2}$. This inequality suggests that the growing of mass resolution is very effective to force convergence for $\ell \gtrsim 1200$ and less effective for greater angular scales.

\subsection{Contrasting AP3M and PM simulations of the RS effect}

We next compare RSSs generated with the AP3M $N$-body code used here, with the RSSs computed in paper I using a PM code (full code details can be found in Quilis, Ibáñez \& Sáez 1998). The RS power spectra corresponding to three simulations were presented in paper I. These spectra are shown by the discontinuous lines in Fig. 8. The dotted line corresponds to a flat universe with $\Omega_{\mathrm{d}}=$ $0.27, h=0.71, \sigma_{8}=0.93, n_{\mathrm{s}}=1$ (Harrison-Zel'dovich spectrum), simulated using a PM code with $L_{\text {box }}=256 \mathrm{Mpc}, N_{\mathrm{c}}=512$, $N_{\mathrm{p}}=512^{3}, N_{\text {dir }}=256, z_{\text {in }}=5.2$, and $L_{\max }=42 h^{-1} \mathrm{Mpc}$. Since the effective resolution, $E_{\text {res }}$, of the PM code is two cells, this simulation has $E_{\text {res }}=0.5 h^{-1}$ Mpc. A photon step, $\Delta_{\mathrm{ps}}=0.25 h^{-1} \mathrm{Mpc}$ was found to be adequate to take advantage of this effective resolution (smaller values lead to very similar results). The other two PM RSSs were generated by varying the number of cells and particles: $N_{\mathrm{c}}=256, N_{\mathrm{p}}=256^{3}$, effective resolution $1 \mathrm{Mpc}$ (dashed line); and $N_{\mathrm{c}}=128, N_{\mathrm{p}}=128^{3}$ effective resolution $2 \mathrm{Mpc}$. The three PM RSSs (discontinuous lines) show that the greater the PM resolution the smaller the RS signal. This fact and the comparison of the three discontinuous lines suggest a possible convergence towards $\Delta_{\mathrm{T}}$ val-

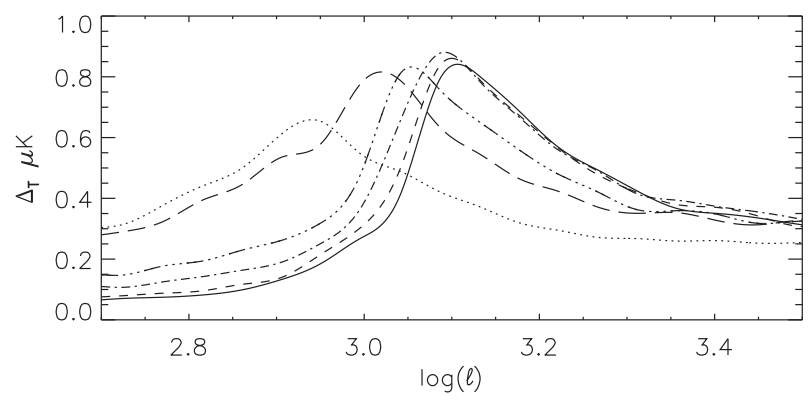

Figure 9. Quantity $\Delta_{\mathrm{T}}$ as a function of $\log (\ell)$ for varying initial redshift. Solid, dashed, dot-dashed, triple-dot-dashed, long-dashed, and dotted lines correspond to $z_{\text {in }}=30,25,20,15,10$, and 6 , respectively. The remaining parameters are the same as those of the reference RSSs.

ues smaller than those of the dotted line; however, more detailed simulations are needed to confirm or refute this convergence.

To examine the impact of submesh-scale resolution on the RS signal, a new AP3M simulation of the RS effect was conducted. In this RSS, the parameters had the same values as in the above PM simulations, except for $L_{\text {box }}=512 h^{-1} \mathrm{Mpc}, N_{\mathrm{p}}=256^{3}$, $N_{\mathrm{c}}=1024$, and $S_{\mathrm{p}}=50 \mathrm{~h}^{-1} \mathrm{kpc}$, other parameters have the same values as in the reference RSSs. In this case, the effective resolution is $\sim 250 h^{-1} \mathrm{kpc}$, which is greater than the best resolution of the three PM simulations. For $E_{\text {res }} \sim 250 h^{-1} \mathrm{Kpc}$, we may use the integration step $\Delta_{\mathrm{ps}}=100 \mathrm{~h}^{-1} \mathrm{kpc}$ (see Section 4.3). We emphasize that the same ray-tracing procedure, described in Section 3.2, has been used to deal with photon propagation in both PM- and AP3Msimulated periodic universes. The solid line of Fig. 8 represents the angular power spectrum of the AP3M simulation, which may be compared with the spectra displayed in the three discontinuous lines (PM RSSs). This comparison leads to the conclusion that the dotted and dashed lines approximately indicate the region where the RS power spectrum lies; however, the shape of these lines is not similar to that of the solid line (AP3M simulation). Finally, the solid line is not below the dotted line, which means that the aforementioned convergence suggested by the PM spectra (discontinuous lines) is only apparent. It is a consequence of using low-resolution PM simulations.

\subsection{Effect of changing the initial ray-tracing redshift $z_{\text {in }}$}

According to the considerations of Section 2, the RS effect is produced by all non-linear structures. If the full effect is to be estimated with our AP3M simulations, the initial redshift cannot be $z_{\text {in }}=5.2$, as it was for most of the simulations in paper I or $z_{\text {in }}=10$ as in Cai et al. (2010). With these choices, all the strongly non-linear structures are likely considered, but the RS effect due to slightly and mildly non-linear inhomogeneities evolving at higher redshifts is missed.

To find out how the RS signal is accumulated, we have obtained the $C_{\ell}$ coefficients, for the reference RSSs, with the following values of $z_{\text {in }}: 6,10,15,20,25$, and 30 . The six resulting spectra are displayed in Fig. 9. It is evident that the spectra corresponding to $z_{\text {in }}=25$ and 30 are very similar, which means that there are no significant contributions to the total RS signal at $z_{\text {in }}>25$ (almost linear structure evolution). The spectra of Fig. 9 appears to converge for a value of $z_{\text {in }}$ to 25 and motivate the choice of this value for the reference RSSs. As may be seen in Figs 1-9, the power of the RS signal has a maximum, which for $z_{\text {in }}=30$, corresponds to $\ell \simeq 1280$, and the power is greater than about $0.6 \mu \mathrm{K}$ in the $\ell$ interval (1150, 


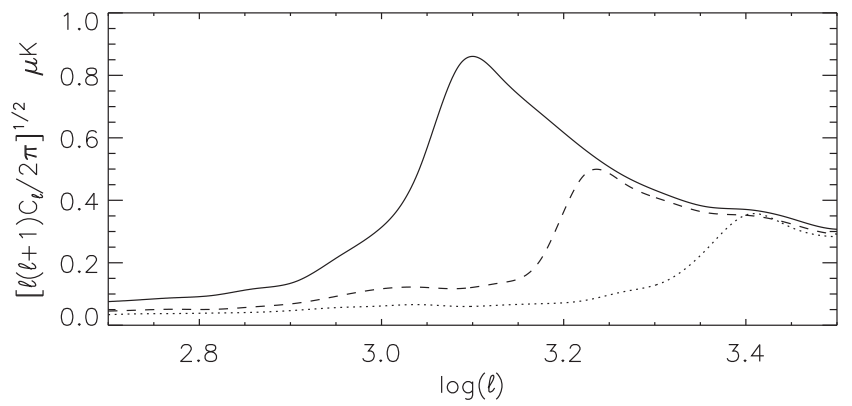

Figure 10. $\Delta_{\mathrm{T}}$ power spectrum in terms of $\log (\ell)$ for different values of the potential cutoff length. All the parameters have the same values as in the reference RSSs, except for the cutoff length $L_{\max }$. Solid, dashed, and dotted curves correspond to $L_{\max }=42 h^{-1} \mathrm{Mpc}, L_{\max }=30 h^{-1} \mathrm{Mpc}$ and $L_{\max }=21 h^{-1} \mathrm{Mpc}$, respectively.

1600). Could we detect a signal so weak in this $\ell$ interval? This important question is discussed in the next section.

\subsection{Effect of changing the cutoff length $L_{\max }$}

AP3M HYDRA simulations with high resolutions are not necessary to describe the RS effect produced by scales greater than, e.g. $42 h^{-1}$ Mpc. PM simulations should be sufficient to superimpose the RS effects of these scales, since the PP corrections essentially affect much smaller spatial scales. Since these PM simulations would have a lower computational cost, they could be run in very large boxes from high initial redshifts; evidently, at high enough redshifts, the Zel'dovich approximation, or some appropriate generalization (see Matarrese et al. 1992 and references therein), should be sufficient to deal with the RS effect due to comoving scales $L>42 h^{-1}$ Mpc. In these PM simulations, scales smaller than $42 h^{-1} \mathrm{Mpc}$ would be removed (from the peculiar potential $\phi$ ) to calculate the RS effect complementary of that estimated in this paper for scales $L<L_{\text {max }}=42 h^{-1} \mathrm{Mpc}$.

Note also that for a galaxy cluster having a dark matter density profile with virial radius $S \sim 2 h^{-1} \mathrm{Mpc}$, the profile of $\phi$ has a much greater size involving significant spatial scales of a few tens of megaparsecs; hence, if the cutoff on $\phi$ is performed, for $L_{\max }=$ $42 h^{-1} \mathrm{Mpc}$, the galaxy cluster contribution to the RS signal will be fully included in our estimates.

Cutoff lengths between 42 and $72 h^{-1}$ Mpc have been also considered, and the resulting spectra are displayed in the discontinuous lines of Fig. 1. Cutoffs at $L_{\max }>72 h^{-1} \mathrm{Mpc}$ should not be performed in reference RSSs with $L_{\text {box }} / f=512 h^{-1} \mathrm{Mpc}$ and $z_{\text {in }}=$ 25 (next section for further discussion).

Based upon the preceding considerations, we have chosen $L_{\max }=$ $42 h^{-1} \mathrm{Mpc}$ as the cutoff length of the reference RRSs. Two other RSSs with the same parameters as those of the reference, but with cutoff lengths $L_{\max }=30 h^{-1} \mathrm{Mpc}$ and $L_{\max }=21 h^{-1} \mathrm{Mpc}$, have been also examined to build up Fig. 10. From the power spectra obtained for the $L_{\max }$ values of Fig. 10, the contribution of different ranges of non-linear scales to the RS effect may be estimated. For scales $L<L_{\max }=21 h^{-1} \mathrm{Mpc}$, the RS signal is small and it takes on its maximum value at $\ell \simeq 2500$. This signal should be essentially due to strongly non-linear structures as clusters. The peak around $\ell=1250$ is mainly produced by mildly non-linear structures and clusters, whose potentials $\phi$ involve scales belonging to the $L$ interval $(21,42) h^{-1} \mathrm{Mpc}$. Similarly, the contributions to the RS effect due to the scales in the $L$ intervals $(42,52) h^{-1} \mathrm{Mpc},(52$,
62) $h^{-1} \mathrm{Mpc}$, and $(62,72) h^{-1} \mathrm{Mpc}$ follow from the discontinuous lines of Fig. 1.

\section{CONCLUSIONS AND DISCUSSION}

Our reference RSSs have allowed us to estimate the angular power spectrum of the RS effect produced by scales $L \leq 42 h^{-1} \mathrm{Mpc}$. This is a part of the total RS effect which we denote in the RS-42 effect.

We have shown that, for an ensemble created from a large enough number of reference RSSs $(\geq 6$ ), a reliable estimate of the RS-42 angular power spectrum may be obtained (hereafter $R S-42$ reference spectrum). In particular, it has been verified that the choice of parameters for the reference RSSs is robust. We have fixed $L_{\max }=42 h^{-1} \mathrm{Mpc}$ and show that spectrum is almost the same for any $z_{\mathrm{f}} \leq 0.5$. We have performed an extensive set of parameter sensitivity studies and conclude that: (i) for a smoothing parameter $S_{\mathrm{p}}$ satisfying $25 h^{-1} \leq S_{\mathrm{p}} \leq 75 h^{-1} \mathrm{kpc}$, the RS-42 angular power spectrum is almost indistinguishable from the RS-42 reference spectrum ( $S_{\mathrm{p}}=50 \mathrm{~h}^{-1} \mathrm{kpc}$ ); (ii) simulations with box sizes in the interval (2561 024) $f h^{-1}$ Mpc lead to comparable RS-42 spectra if the number of particles is appropriately chosen (same particle number density); (iii) for $N_{\mathrm{p}}$ values of $256^{3}$ (reference RSSs) and $512^{3}$, and $L_{\text {box }}=512 h^{-1} \mathrm{Mpc}$, the resulting $C_{\ell}$ coefficients are very similar. As $N_{\mathrm{p}}$ increases, there is convergence towards an angular power spectrum very similar to the RS-42 reference spectrum; and, (iv) as the $z_{\text {in }}$ redshift increases in the interval [6,30], the angular power spectrum changes, taking very similar values for $z_{\text {in }}=$ 25 (reference RSSs) and $z_{\text {in }}=30$, which shows again convergence towards a spectrum very similar to the RS-42 reference spectrum.

The redshift intervals in which the RS-42 effect is produced are studied in Sections 4.2 and 4.6. This analysis allows us to discuss if the detection of the RS-42 effect is feasible, but we will start by discussing the well-known L-ISW detection method.

In the flat $\Lambda$ CDM model, it is well known that the L-ISW effect is vanishing (negligible) for $\Omega_{\Lambda} \sim 0$. By exploiting this result, Crittenden \& Turok (1996) proposed a method to look for a possible relevant cosmological constant. At that time, before the analysis of the supernova Ia luminosities (Riess et al. 1998; Perlmutter et al. 1999) suggesting an accelerating universe, and after CMB anisotropy detection $(\ell \leq 20)$ with COBE (Cosmic Background Explorer; Smoot et al. 1992), the observational evidence supporting a significant cosmological constant was both scarce and weak and, consequently, the proposal by Crittenden \& Turok (1996) was very timely. These authors took into account that the L-ISW effect is produced at low redshifts $(z \leq 1)$ and is mainly relevant for $\ell \leq 100$ (see Fig. 1), which means that it is produced by the time-varying gravitational potential of large-scale structures. As a result, they concluded that, in the presence of a cosmological constant, there must be a cross-correlation between the CMB temperature distribution (COBE data), which includes the L-ISW effect, and tracers of large-scale structure at $z \leq 1$ (X-ray ROSAT survey data at that time). In short, following Crittenden \& Turok (1996), researchers should look for these correlations to identify a non-zero cosmological constant.

Today's situation is different. As noted in Section 1, current observational data lead to predictions for the cosmological parameters in the context of $\Lambda \mathrm{CDM}$ with small $1 \sigma$ uncertainties: e.g. one finds $\Omega_{\Lambda}=0.692 \pm 0.010$ (see last column of table 5 in Planck Collaboration XVI 2014a). This means that current observational data are not compatible with $\Omega_{\Lambda}=0$ in the $\Lambda$ CDM model and, consequently, one can state that there is an L-ISW effect and 

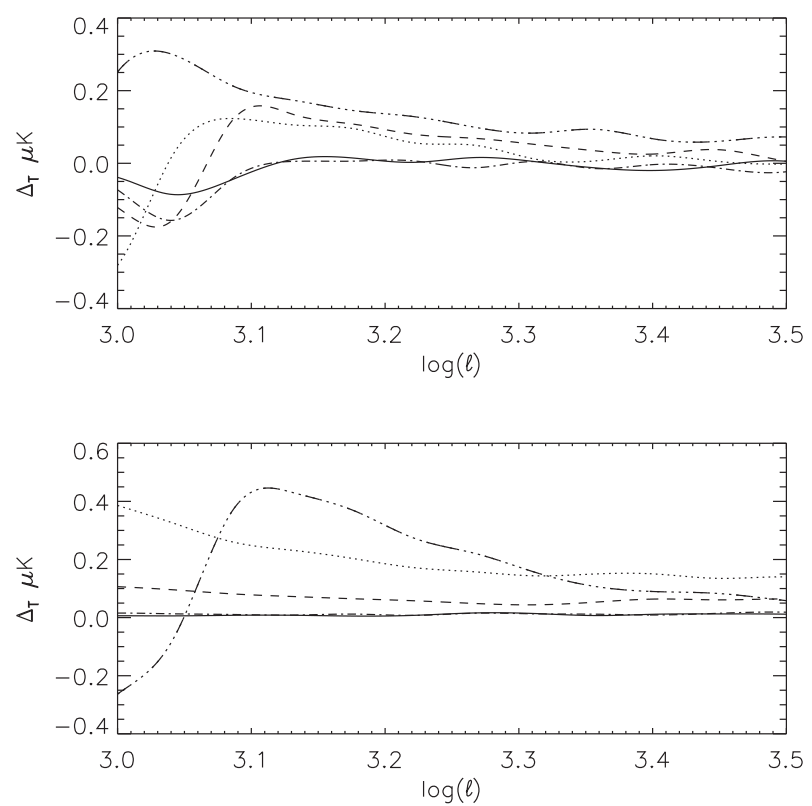

Figure 11. Contributions to $\Delta_{\mathrm{T}}$, produced in various redshift intervals, as functions of $\log (\ell)$. Calculations are based on the reference RSSs. Top panel: triple-dot-dashed, dotted, dashed, dot-dashed, and solid lines correspond to the intervals $[6,10],[10,15],[15,20],[20,25]$, and [25,30], respectively. Bottom panel: triple-dot-dashed, dotted, dashed, dot-dashed, and solid curves are the contributions in the intervals $[6,30],[4,6],[2,4],[1,2]$, and $[0.5,1]$, respectively.

cross-correlations should exist between the Planck CMB temperature distribution and tracers of the dark matter distribution on large scales for $z \leq 1$. These correlations have been predicted in the context of the $\Lambda \mathrm{CDM}$ model and, for appropriate tracers (Planck Collaboration XVII 2014b), they have been recently detected with rather high statistical significances. Previous detections are numerous, see also Planck Collaboration XVII (2014b) for an exhaustive list.

In any cosmological model which predicts time variations of the potential, $\phi$, produced by linear structures, there will be an L-ISW effect given by equation (6); the $\Lambda \mathrm{CDM}$ model is only a particular case. In different cosmological models, $\partial \phi / \partial \eta$ may contribute significantly to the integral of equation (6) in different redshift interval $\left[z_{1}, z_{2}\right](z \leq 1$ for $\Lambda \mathrm{CDM})$ and, consequently, one must look for cross-correlations with tracers of large-scale structure located between redshifts $z_{1}$ and $z_{2}$. This is only possible with good enough data. In each model, specific cross-correlations may be predicted and, then, by comparing predictions and detection (if viable), the model could be either confirmed or ruled out. According to Planck Collaboration XVII (2014b), some detected cross-correlations are compatible with the $\Lambda \mathrm{CDM}$ predictions; nevertheless, other models may also be admissible.

Let us now consider the RS-42 effect. As has been shown in previous sections, contributions to this effect arise from various scales evolving in various redshift intervals; these scales contribute to the Fourier expansion of the peculiar gravitational potential, $\phi$, corresponding to non-linear cosmological structures. In view of this, we will try to answer the following question: is the detection of the RS-42 effect possible by looking for correlations between Planck CMB temperature maps and tracers of non-linear structure? To answer, we may refer to the results in Fig. 11. In particular, the solid and dot-dashed lines in the bottom panel of Fig. 11 show that the RS-42 effect produced at $z \leq 2$ is completely negligible, and
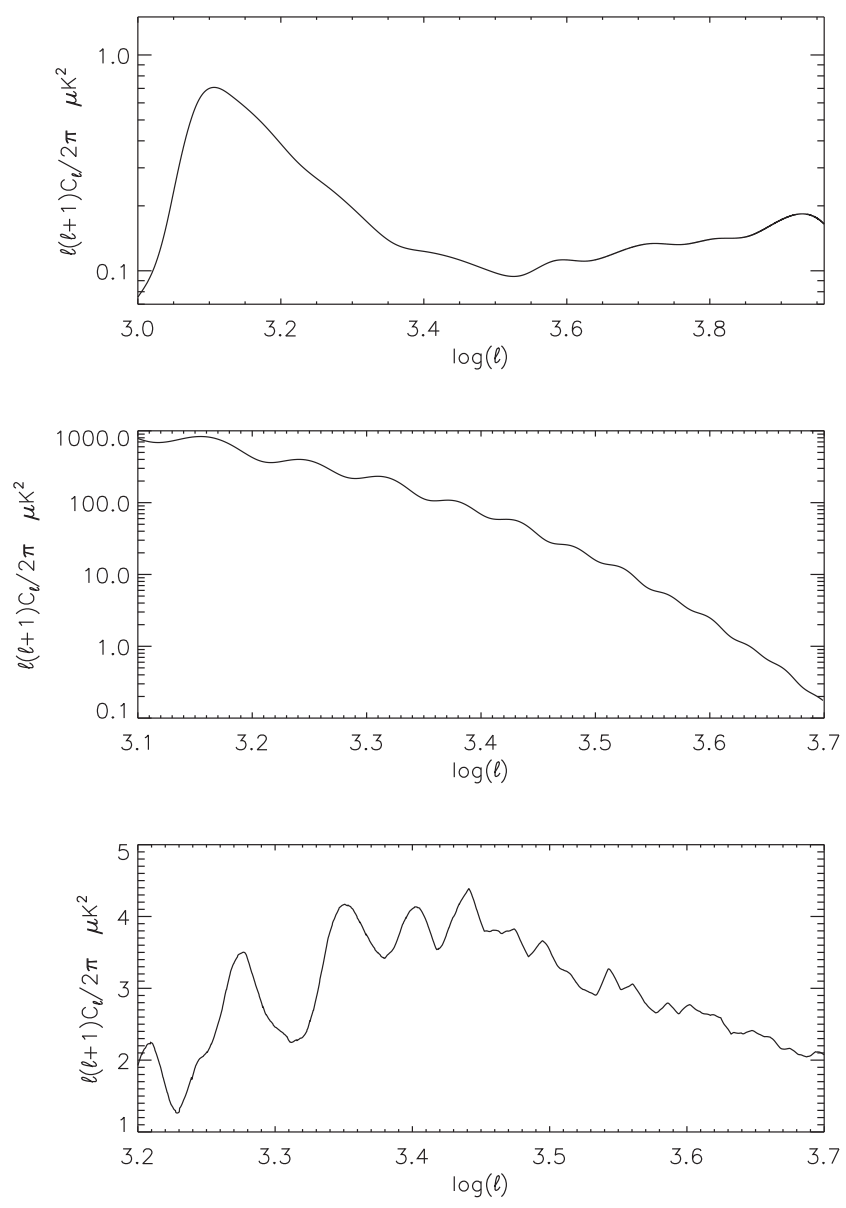

Figure 12. Quantity $\ell(\ell+1) C_{\ell} / 2 \pi \equiv \Delta_{\mathrm{T}}^{2}$ in terms of $\log (\ell)$. Curves in the top, middle, and bottom panels display the spectra corresponding to RS-42, primary anisotropy, and weak lensing, respectively.

is too small for $2 \leq z \leq 4$. Hence, one would need to look for cross-correlations between $\mathrm{CMB}$ data and tracers of non-linear structures at $z>4$ (see all the curves in Fig. 11). It is not possible to recover these cross-correlations since there are no suitable observational maps of any tracer at these high redshifts (see Section 2.2 and fig. 2 in Ade et al. 2014b) and, moreover, as discussed in the next paragraphs, the RS-42 imprint on the measurable CMB temperature distribution is too small to be directly observed.

Fig. 12 (top panel) shows that around the peak of the RS-42 spectrum, which occurs at $\log \ell \sim 3.1(\ell \sim 1250)$, the amplitude of $\left(\Delta_{\mathrm{T}}^{2}\right)_{R S-42}$ is close to $0.77 \mu K^{2}$. The power of the primary anisotropy at this scale (for the model described in Section 1) is $\left(\Delta_{\mathrm{T}}^{2}\right)_{\mathrm{PR}} \sim 800 \mu K^{2}$ (middle panel). This means that, at the peak, the $C_{\ell}$ coefficients of the primary anisotropy are around 1100 times greater than those of the RS-42 effect, which would thus clearly be unobservable.

Fig. 12 also shows that the situation becomes better for larger $\ell$ values; e.g. for $\log \ell=3.65$, the power of the primary anisotropy falls to $\left(\Delta_{\mathrm{T}}^{2}\right)_{\mathrm{PR}} \sim 0.7 \mu K^{2}$ (middle), whereas the power of the RS42 signal is $\left(\Delta_{\mathrm{T}}^{2}\right)_{R S-42} \sim 0.1 \mu K^{2}$. The ratio $\left(\Delta_{\mathrm{T}}^{2}\right)_{\mathrm{PR}} /\left(\Delta_{\mathrm{T}}^{2}\right)_{R S-42}$ fast decreases, but $\left(\Delta_{\mathrm{T}}^{2}\right)_{\mathrm{RS}-42}$ remains close to $\sim 0.1 \mu K^{2}$ and, evidently, this value is too small to be measured on account of the attainable precision of realistic experiments. Moreover, in the $\log \ell$ interval $[3.5,3.7]$, the main hindrance to detection is not the primary anisotropy, which reaches very small values, but weak lensing, Sunyaev-Zel'dovich (thermal and kinematic), contaminant 
foregrounds (cosmic infrared background, radio galaxies) and noise. The bottom panel exhibits the weak lensing power numerically estimated in Fullana et al. (2010), where it is observed that, for $\log \ell$ $=3.65$, one has $\left(\Delta_{\mathrm{T}}^{2}\right)_{\mathrm{WL}} \sim 2.4 \mu K^{2}$. The coupling between the kinematic and thermal Sunyaev-Zel'dovich components would be responsible for a power $\left(\Delta_{\mathrm{T}}^{2}\right)_{\mathrm{SZ}} \sim 10 \mu K^{2}$ (George et al. 2015). The total power at these angular scales would be a few tens of $\mu K^{2}$ (see fig. 12 in Das et al. 2014) and, consequently, a signal as small as $0.1 \mu K^{2}$ cannot be isolated from the above superimposition of effects $\left(\sim 30 \mu K^{2}\right)$, whose uncertainties are much bigger than the RS-42 signal.

Finally, the top panel of Fig. 12 also shows that, at $\log \ell \simeq$ 3.55 , the RS-42 power starts to increase. This upturn could be caused by Poisson noise due to discretization, however, it does not seem to be the case, since the growth is not triggered when the resolution decreases (see Fig. 7). Further investigation of this point is necessary.

In Fig. 10, the RS spectra due to scales smaller than $30 h^{-1} \mathrm{Mpc}$ (RS-30) and $21 h^{-1} \mathrm{Mpc}$ (RS-21) are presented together with the RS-42 spectrum; moreover, the broken lines in Fig. 1 give us, from bottom to top, the RS-42, RS-52, RS-62, and RS-72 spectra. In these two figures, we see that, as $L_{\max }$ increases and the RS effect of larger scales is added, the peak of the RS spectrum grows and moves to smaller $\ell$. For scales larger than $72 h^{-1} \mathrm{Mpc}$, this trend must evolve in such a way that the RS and L-ISW spectra connect to give a unique spectrum, however, as discussed below, our methods, based on high-resolution $\mathrm{N}$-body simulations, are not the most suitable to achieve this connection.

In the reference RSSs, the initial redshift must be greater than $\sim 25$ (see Fig. 9). This is easily understood by noting that these simulations involve spatial $\phi$-scales with $L<42 h^{-1} \mathrm{Mpc}$. Some of these $\phi$-scales, e.g. those close to $42 h^{-1} \mathrm{Mpc}$, correspond to density contrasts evolving as weakly or mildly non-linear structures and, structures of this kind (less extended than the $\phi$-profiles) are already contributing to the RS effect at $z \sim 25$. A smaller initial redshift may be used to study the RS effect due to strongly non-linear structures, namely, for a cutoff $L_{\max }<42 h^{-1} \mathrm{Mpc}$ (see Fig. 10 and comments in Section 4.7). However, for cutoffs with $L_{\max } \gg 42 h^{-1} \mathrm{Mpc}$, which would be necessary to connection the L-ISW and RS effects, the largest scales would be present at $z_{\text {in }}>25$, perhaps at $z_{\text {in }}>50$ which is the initial redshift of our $N$-body simulations. Finally, if too large $\phi$-scales are considered by choosing $L_{\max } \gg 42 h^{-1} \mathrm{Mpc}$, even for the preferred directions, photons might cross the same large-scale region various times in our simulated periodic universe and, consequently, RS magnification would arise. Much more extended boxes would be necessary to properly perform cutoffs with $L_{\max } \gg 42 h^{-1} \mathrm{Mpc}$, entailing a very high computational cost in order to maintain an admissible level of accuracy.

We have accurately estimated the RS-42 spectrum and, then, to discuss the connection between the L-ISW and RS spectra, have considered the RS effect due to weakly and mildly non-linear scales larger than $42 h^{-1} \mathrm{Mpc}$ but smaller than $72 h^{-1} \mathrm{Mpc}$. To achieve the connection, scales larger than $72 h^{-1} \mathrm{Mpc}$ must be taken into account; this might be done in future by using PM codes combined with appropriate approximations (see Section 4.7). According to Fig. 1 (discontinuous lines), the RS contributions of scales between 42 and $72 h^{-1} \mathrm{Mpc}$ are smaller than $\sim 2 \mu \mathrm{K}$ and, moreover, these contributions appear in the $\ell$ range where the primary anisotropy is strongly dominant; hence, after the above discussion of the RS-42 observability, it follows that RS-72 would be neither observable.
Finally, we comment on possible baryon contributions to the RS power. As has been discussed earlier, most of the RS effect is produced, at redshifts $z>4$, by spatial scales contributing significantly to the Fourier expansion of $\phi$. According to Fig. 10, these scales are larger than $\sim 20 h^{-1} \mathrm{Mpc}$; in contrast, however, WL is produced at much lower redshifts by smaller scales relevant to the expansion of the transverse gradient of $\phi$. The contributions of baryons to the RS power depend on the modifications that this scarce kind of matter causes on $\partial \phi / \partial t$ at redshifts $z>4$. At these redshifts, subdominant baryon matter is falling in the potential wells of dark matter to form protoclusters; but the baryons have not yet collapsed to form central cores with relevant feedback, cooling, and other effects, which are already ongoing at lower redshifts where WL is produced. This suggests that the presence of baryons must affect RS power less than it does WL power. While hydrodynamic simulations, such as those conducted in Jing et al. (2006), Semboloni et al. (2011), and Osato et al. (2015), could be used to properly estimate the RS effect due to baryons, the above analysis suggests this effort is not necessary, as a contribution to the RS power, as large as the greatest baryon contributions to WL (about 10 per cent), would not affect either our main conclusions or the above discussion.

\section{ACKNOWLEDGEMENTS}

This work has been supported by the Spanish Ministry of Economía y Competitividad, MICINN-FEDER project FIS2015-64552-P. Calculations were carried out at the Centre de Càlcul de la Universitat de València. RJT thanks NSERC, the Canada Research Chairs Program, Canada Foundation for Innovation and the Nova Scotia Research and Innovation Trust for support. HMPC thank NSERC for support.

\section{REFERENCES}

Abbott L. F., Schaefer R. K., 1986, ApJ, 308, 546

Antón L., Cerdá-Durán P., Quilis V., Sáez D., 2005, ApJ, 628, 1

Bardeen J. M., 1980, Phys. Rev. D, 22, 1882

Barreira A., Llinares C., Bose S., Li B., 2016, J. Cosmol. Astropart. Phys., 05,001

Bond J. R., Efstathiou G., 1987, MNRAS, 226, 655

Cai Y.-C., Cole S., Jenkins A., Frenk C. S., 2010, MNRAS, 407, 201

Carbone C., Springel V., Baccigalupi C., Bartelmann M., Matarrese S., 2008, MNRAS, 388, 1618

Critteden R. G., Turok N., 1996, Phys. Rev. Lett., 76, 575

Das S. et al., 2014, J. Cosmol. Astropart. Phys., 04, 014

Efstathiou G., Davis M., White S. D. M., Frenk C., 1985, ApJS, 57, 241

Fullana M. J., Arnau J. V., Thacker R. J., Couchman H. M. P., Sáez D., 2010, ApJ, 712, 367

George E. M. et al., 2015, ApJ, 799, 177

Hamana T., Colombi S., Suto Y., 2001, A\&A, 367, 18

Hernquist L., Katz N., 1989, ApJS, 70, 419

Hilbert S., Hartlap J., White S. D. M., Schneider P., 2009, A\&A, 499, 31

Hockney R. W., Eastwood J. W., 1988, Computer Simulations Using Particles. CRC Press, Boca Raton, FL

Hu W., White M., 1997, Phys. Rev. D, 56, 596

Jain B., Seljak U., White S., 2000, ApJ, 530, 547

Jing Y. P., Zhang P., Lin W. P., Gao L., Springel V., 2006, ApJ, 640, L119

Lewis A., Challinor A., Lasenby A., 2000, ApJ, 538, 473

Ma C. P., Bertschinger E., 1995, ApJ, 455, 7

Matarrese S., Lucchin F., Moscardini L., Sáez D., 1992, MNRAS, 259, 437

Moore B., Governato F., Quinn T., Stadel J., Lake G., 1998, ApJ, 499, L5

Osato K., Shirasaki M., Yoshida N., 2015, ApJ, 806, 186

Peebles P. J. E., 1980, The Large Scale Structure of the Universe. Princeton Univ. Press, Princeton, NJ 
Perlmutter S. et al., 1999, ApJ, 517, 565

Planck Collaboration XVI, 2014a, A\&A, 571, A16

Planck Collaboration XVII, 2014b, A\&A, 571, A19

Pratt W. K., 2013, Introduction to Digital Image Processing. CRC Press, Boca Raton, FL

Puchades N., Fullana M. J., Arnau J. V., Sáez D., 2006, MNRAS, 370, 1849

Quilis V., Ibáñez J. M., Sáez D., 1998, ApJ, 502, 518

Rees M. J., Sciama D. W., 1968, Nature, 217, 511

Riess A. G. et al., 1998, AJ, 116, 1009

Sachs R. K., Wolfe A. M., 1967, ApJ, 147, 73

Sáez D., Holtmann E., Smoot G. F., 1996, ApJ, 473, 1

Sáez Milán D. P., Puchades N., Arnau i Córdoba J. V., Fullana M. J., 2006, in de Zotti G., eds., Proc. Sci., Ray-tracing through N-body simulations and CMB anisotropy estimations. SISSA, Trieste, PoS(CMB2006)058

Sato M., Hamana T., Takahashi R., Takada M., Yoshida N., Matsubara T., Sugiyama N., 2009, ApJ, 701, 945
Seljak U., Zaldarriaga M., 1996, ApJ, 469, 437

Semboloni E., Hoekstra H., Schaye J., van Daalen M. P., McCarthy I. G., 2011, MNRAS, 417, 2020

Smoot G. F. et al., 1992, ApJ, 396, L1

Springel V., White M., Hernquist L., 2001, ApJ, 549, 681

Springel V. et al., 2005, Nature, 435, 629

Thacker R. J., Couchman H. M. P., 2006, Comput. Phys. Commun., 174, 540

Tuluie R., Laguna P., Anninos P., 1996, ApJ, 463, 15

Van Waerbeke L., Hamana T., Scoccimarro R., Colombi S., Bernardeau F., MNRAS, 322, 918

White M., Hu W., 2001, ApJ, 537, 1

This paper has been typeset from a $\mathrm{T}_{\mathrm{E}} \mathrm{X} / \mathrm{L} \mathrm{T} \mathrm{E} \mathrm{X}$ file prepared by the author. 